\title{
Asymptotics for Products of a Random Number of Partial Sums
}

by

\author{
Marcin PRZYSTALSKI \\ Presented by Stanistaw KWAPIEŃ
}

Summary. We consider products of a random number of partial sums of independent, identically distributed, positive square-integrable random variables. We show that the distribution of these products is asymptotically lognormal.

1. Introduction. Let $\left(X_{n}\right)_{n \in \mathbb{N}}$ be a sequence of independent and identically distributed (iid), positive and square-integrable random variables. Let us define the partial sums

$$
S_{k}=\sum_{i=1}^{k} X_{i}, \quad k=1,2, \ldots
$$

Asymptotics for products of such sums have been studied by several authors. Arnold and Villaseñor [1] obtained a limit theorem for a sequence $\left(X_{n}\right)$ of iid exponential variables with mean 1 ,

$$
\frac{\sum_{k=1}^{n} \log \left(S_{k}\right)-n \log (n)+n}{\sqrt{2 n}} \stackrel{d}{\rightarrow} \mathcal{N}
$$

as $n \rightarrow \infty$, where $\stackrel{d}{\rightarrow}$ stands for convergence in distribution and $\mathcal{N}$ is a standard normal random variable. Their result can be equivalently stated, in terms of products of partial sums, as

$$
\left(\prod_{k=1}^{n} \frac{S_{k}}{k}\right)^{1 / \sqrt{n}} \stackrel{d}{\rightarrow} e^{\sqrt{2} \mathcal{N}} .
$$

2000 Mathematics Subject Classification: Primary 60F05.

Key words and phrases: central limit theorem, lognormal distribution, product of sums of iid random variables. 
In [6] Rempała and Wesołowski proved, without assuming any particular distribution for $X_{i}$ 's, that if $\left(X_{n}\right)$ is a sequence of iid positive square-integrable random variables with $\mu=E\left(X_{1}\right), \sigma^{2}=\operatorname{Var}\left(X_{1}\right)>0$ and variation coefficient $\gamma=\sigma / \mu$ then as $n \rightarrow \infty$ we have

$$
\left(\prod_{k=1}^{n} \frac{S_{k}}{k \mu}\right)^{\frac{1}{\gamma \sqrt{n}}} \stackrel{d}{\rightarrow} e^{\sqrt{2} \mathcal{N}} .
$$

They also extended (2) to U-statistics. The result (2) was further generalized in $[3,5,7]$. In the latter paper, the authors applied their generalization to calculating the asymptotic distribution of Wishart determinants.

The purpose of this note is to generalize (2) to the case of products of a random number of partial sums. Such products, with a slightly different normalization, have also been analyzed in [4].

\section{Main result}

TheOREM 1. Let $\left(X_{n}\right)_{n \in \mathbb{N}}$ be a sequence of iid, positive and squareintegrable random variables with mean $\mu=E\left(X_{1}\right), \sigma^{2}=\operatorname{Var}\left(X_{1}\right)>0$, variation coefficient $\gamma=\sigma / \mu$ and $S_{k}=\sum_{i=1}^{k} X_{i}, k=1,2, \ldots$ Further, let $N_{n}$ denote a positive integer-valued random variable such that $N_{n} / n$ converges as $n \rightarrow \infty$ in probability to a constant $c>0$. Then

$$
\left(\prod_{k=1}^{N_{n}} \frac{S_{k}}{k \mu}\right)^{\frac{1}{\gamma \sqrt{N_{n}}}} \stackrel{d}{\rightarrow} e^{\sqrt{2} \mathcal{N}} .
$$

We prepare the proof of Theorem 1 with two lemmas.

Lemma 2. Under the assumptions of Theorem 1,

$$
\frac{1}{\gamma \sqrt{2 n}} \sum_{k=1}^{n}\left(\frac{S_{k}}{k \mu}-1\right) \stackrel{d}{\rightarrow} \mathcal{N}
$$

Proof. For the proof see [6].

Lemma 3. Under the assumptions of Theorem 1,

$$
\frac{1}{\gamma \sqrt{2 N_{n}}} \sum_{k=1}^{N_{n}}\left(\frac{S_{k}}{k \mu}-1\right) \stackrel{d}{\rightarrow} \mathcal{N}
$$

The proof of Lemma 3 is based on the method described in [8].

Proof. Let $Y_{i}=\left(X_{i}-\mu\right) / \sigma, i=1,2, \ldots, \widetilde{S}_{k}=\sum_{i=1}^{k} Y_{i}, k=1,2, \ldots$. Then (4) can be expressed as

$$
\frac{1}{\sqrt{2 N_{n}}} \sum_{k=1}^{N_{n}} \frac{\widetilde{S}_{k}}{k} \stackrel{d}{\rightarrow} \mathcal{N} .
$$


Let $\varepsilon>0$ be an arbitrary number. By the convergence of $N_{n}$, we can choose $n_{1}>0$ such that for $n \geq n_{1}$,

$$
P\left(\left|N_{n}-n c\right|>c n \varepsilon\right) \leq \varepsilon .
$$

It is clear that

$$
P\left(\frac{1}{\sqrt{2 N_{n}}} \sum_{k=1}^{N_{n}} \frac{\widetilde{S}_{k}}{k}<x\right)=\sum_{m=1}^{\infty} P\left(\frac{1}{\sqrt{2 m}} \sum_{k=1}^{m} \frac{\widetilde{S}_{k}}{k}<x, N_{n}=m\right) .
$$

From (5) and (6) we see that for $n \geq n_{1}$,

$$
\left|P\left(\frac{1}{\sqrt{2 N_{n}}} \sum_{k=1}^{N_{n}} \frac{\widetilde{S}_{k}}{k}<x\right)-\sum_{|m-c n|<c n \varepsilon} P\left(\frac{1}{\sqrt{2 m}} \sum_{k=1}^{m} \frac{\widetilde{S}_{k}}{k}<x, N_{n}=m\right)\right| \leq \varepsilon .
$$

If we define $M_{1}=[(1-\varepsilon) c n]$ and $M_{2}=[(1+\varepsilon) c n]$, where $[\ldots]$ is the integer part, then for $|m-c n|<c n \varepsilon$ we get

$$
P\left(\frac{1}{\sqrt{2 m}} \sum_{k=1}^{m} \frac{\widetilde{S}_{k}}{k}<x, N_{n}=m\right) \leq P\left(\sum_{k=1}^{M_{2}} \frac{\widetilde{S}_{k}}{k}<x \sqrt{2 M_{2}}+\rho, N_{n}=m\right),
$$

where

$$
\rho=\max _{M_{1}<m \leq M_{2}}\left|\sum_{k=M_{1}+1}^{m} \frac{\widetilde{S}_{k}}{k}\right| \text {. }
$$

Similarly

$$
P\left(\frac{1}{\sqrt{2 m}} \sum_{k=1}^{m} \frac{\widetilde{S}_{k}}{k}<x, N_{n}=m\right) \geq P\left(\sum_{k=1}^{M_{1}} \frac{\widetilde{S}_{k}}{k}<x \sqrt{2 M_{1}}-\rho, N_{n}=m\right) .
$$

By the Kolmogorov inequality, we have

$$
\begin{aligned}
P\left(\rho>\left(2 M_{2}\right)^{1 / 2} \delta\right) & \leq P\left(\max _{1 \leq k \leq M_{2}}\left|\widetilde{S}_{k}\right| \max _{1 \leq j \leq\left(M_{2}-M_{1}\right)} \sum_{k=1}^{j} \frac{1}{k+M_{1}}>\left(2 M_{2}\right)^{1 / 2} \delta\right) \\
& =P\left(\max _{1 \leq k \leq M_{2}}\left|\widetilde{S}_{k}\right| \sum_{k=1}^{\left(M_{2}-M_{1}\right)} \frac{1}{k+M_{1}}>\left(2 M_{2}\right)^{1 / 2} \delta\right) \\
& \leq \frac{1}{2 \delta^{2} M_{2}}[\log (1+\varepsilon)]^{2} \operatorname{Var}\left(\widetilde{S}_{M_{2}}\right) .
\end{aligned}
$$

From the assumptions we know that $\operatorname{Var}\left(\widetilde{S}_{M_{2}}\right)=M_{2}$ and finally we get

$$
P\left(\rho>\left(2 M_{2}\right)^{1 / 2} \delta\right) \leq \frac{1}{2 \delta^{2}}[\log (1+\varepsilon)]^{2} \rightarrow 0 \quad \text { as } \varepsilon \rightarrow 0 .
$$


From (7)-(10), it follows that

$$
P\left(\frac{1}{\sqrt{2 N_{n}}} \sum_{k=1}^{N_{n}} \frac{\widetilde{S}_{k}}{k}<x\right) \leq P\left(\frac{1}{\sqrt{2 M_{2}}} \sum_{k=1}^{M_{2}} \frac{\widetilde{S}_{k}}{k}<x+\delta\right)+\mathrm{o}(1)
$$

and

$$
P\left(\frac{1}{\sqrt{2 N_{n}}} \sum_{k=1}^{N_{n}} \frac{\widetilde{S}_{k}}{k}<x\right) \geq P\left(\frac{1}{\sqrt{2 M_{1}}} \sum_{k=1}^{M_{1}} \frac{\widetilde{S}_{k}}{k}<x-\delta\right)+\mathrm{o}(1) .
$$

By Lemma 2 we get the assertion.

Proof of Theorem 1. By Taylor's expansion

$$
\log \left(\frac{S_{k}}{k}\right)=\log \mu+\frac{1}{\mu}\left(\frac{S_{k}}{k}-\mu\right)+\left(\frac{S_{k}}{k}-\mu\right) r\left(\frac{S_{k}}{k}\right),
$$

where $r(x) \rightarrow 0$ as $x \rightarrow \mu$, and we have

$$
\begin{aligned}
\frac{\sum_{k=1}^{N_{n}} \log \left(S_{k} / k\right)-N_{n} \log \mu}{\gamma \sqrt{2 N_{n}}}= & \frac{1}{\gamma \sqrt{2 N_{n}}} \sum_{k=1}^{N_{n}}\left(\frac{S_{k}}{k \mu}-1\right) \\
& +\frac{1}{\gamma \sqrt{2 N_{n}}} \sum_{k=1}^{N_{n}}\left(\frac{S_{k}}{k}-\mu\right) r\left(\frac{S_{k}}{k}\right)
\end{aligned}
$$

By the convergence of $N_{n}$ we have

$$
\frac{1}{\gamma \sqrt{2 N_{n}}} \sum_{k=1}^{N_{n}}\left(\frac{S_{k}}{k}-\mu\right) r\left(\frac{S_{k}}{k}\right) \leq \frac{1}{\gamma \sqrt{2 n c}} \sum_{k=1}^{[n c(1+\varepsilon)]}\left(\frac{S_{k}}{k}-\mu\right) r\left(\frac{S_{k}}{k}\right) .
$$

Because $E\left|X_{1}\right|<\infty$, by the SLLN we have $r\left(S_{k} / k\right) \rightarrow 0$ a.s. From Lemma 3 in [2] it follows that

$$
\begin{aligned}
\sup _{0 \leq \varepsilon \leq 1}\left|\frac{1}{\gamma \sqrt{2 n c}} \sum_{k=1}^{[n c(1+\varepsilon)]}\left(\frac{S_{k}}{k}-\mu\right) r\left(\frac{S_{k}}{k}\right)\right| & \leq \frac{1}{\gamma \sqrt{2 n c}} \sum_{k=1}^{[2 n c]}\left|\frac{S_{k}}{k}-\mu\right|\left|r\left(\frac{S_{k}}{k}\right)\right| \\
& =\mathrm{O}_{\mathbb{P}}(1) .
\end{aligned}
$$

From the above inequality, it follows that

$$
\frac{\sum_{k=1}^{N_{n}} \log \left(S_{k} / k\right)-N_{n} \log \mu}{\gamma \sqrt{2 N_{n}}}=\frac{1}{\gamma \sqrt{2 N_{n}}} \sum_{k=1}^{N_{n}}\left(\frac{S_{k}}{k \mu}-1\right)+\mathrm{O}_{\mathbb{P}}(1) .
$$

By Lemma 3 we get the assertion.

Acknowledgements. I would like to express my gratitude to Prof. Zdzisław Rychlik for reading the first draft of this paper, and the referee for valuable comments and remarks. This work was partially supported by the Polish Ministry of Education and Science grant SPB/COST/KN/DWM 74/2005-2008. 


\section{References}

[1] B. C. Arnold and J. A. Villaseñor, The asymptotic distribution of sums of records, Extremes 1 (1998), 351-363.

[2] K. M. Kosiński, On the functional limits for sums of a function of partial sums, Statist. Probab. Lett. 79 (2009), 1522-1527.

[3] X. Lu and Y. Qi, A note on asymptotic distribution of products of sums, ibid. 68 (2004), 407-413.

[4] T.-X. Pang, Z.-Y. Lin and K.-S. Hwang, Asymptotics for self-normalized random products of sums of i.i.d. random variables, J. Math. Anal. Appl. 334 (2007), 12461259.

[5] Y. Qi, Limit distributions for products of sums, Statist. Probab. Lett. 62 (2003), 93-100.

[6] G. Rempała and J. Wesołowski, Asymptotics for products of sums and U-statistics, Electron. Comm. Probab. 7 (2002), 47-54.

[7] - - - Asymptotics for products of independent sums with an application to Wishart determinants, Statist. Probab. Lett. 74 (2005), 129-138.

[8] A. Rényi, On the asymptotic distribution of the sum of a random number of independent random variables, Acta Math. Acad. Sci. Hungar. 8 (1957), 193-199.

Marcin Przystalski

Biometry Laboratory

Institute of Plant Genetics

Polish Academy of Sciences

Strzeszyńska 34

60-479 Poznań, Poland

E-mail: marprzyst@gmail.com

Received April 24, 2009;

received in final form August 4, 2009 